\title{
Preimplantation genetic diagnosis for $\alpha$-thalassaemia in China
}

\author{
Yan-Wen Xu • Yan-Hong Zeng • Jie Deng • Ying Liu • \\ Ling Gao $\cdot$ Can-Quan Zhou $\cdot$ Guang-Lun Zhuang
}

Received: 24 April 2009 / Accepted: 4 August 2009/Published online: 18 September 2009

(C) The Author(s) 2009. This article is published with open access at Springerlink.com

\begin{abstract}
Purpose To report the usage of PGD for $\alpha$-thalassaemia with the -- ${ }^{\text {SEA }}$ genotype.

Method A PGD protocol using fluorescent gap PCR was performed for 51 cycles on 43 couples with the -- ${ }^{2}$ genotype. Allele drop-out and amplification failure rates were retrospectively analyzed.

Results A total of 472 embryos were biopsied. Amplification was achieved in 390 blastomeres, accounting for an amplification rate of $82.6 \%$. In total, 120 wild-type, 94 heterozygotes and 140 homozygous mutant embryos were diagnosed. The successful diagnosis rate was $75.0 \%$. The ADO rate in 49 blastomeres from six donated embryos was $16.4 \%$. One hundred and fifty four embryos were transferred, resulting in 25 clinical pregnancies with an implantation rate of $24.0 \%$.

Conclusions Single-round fluorescent gap PCR is a feasible and effective strategy in the PGD for $\alpha$-thalassaemia with the -- ${ }^{\text {SEA }}$ genotype.
\end{abstract}

Capsule Single-round fluorescent gap PCR is a feasible and effective strategy in the preimplantation genetic diagnosis (PGD) for $\alpha$-thalassaemia caused by the -- ${ }^{\text {SEA }}$ mutation.

Y.-W. Xu $\cdot$ Y.-H. Zeng $\cdot$ J. Deng $\cdot$ Y. Liu $\cdot$ L. Gao $\cdot$

C.-Q. Zhou $(\bowtie) \cdot$ G.-L. Zhuang

Reproductive Medical Center,

the First Affiliated Hospital of Sun Yat-sen University,

Guangzhou, China

e-mail: zhoucanquan@hotmail.com

Present Address:

C.-Q. Zhou

No 58, Zhongshan Rd 2,

510080 Guangzhou, China
Keywords Preimplantation genetic diagnosis . $\alpha$-thalassaemia $\cdot$ Fluorescent PCR

\section{Introduction}

Thalassaemia is the most common monogenetic hemoglobin disease in Southeast Asia and southern China. Deficiency of the $\alpha$-globin chains results in $\alpha$-thalassaemia. Affected homozygotes of $\alpha$-thalassaemia, Hb Bart's hydrops fetalis, die either in utero in late gestation or within a few minutes after birth. Mothers carrying such hydropic fetuses are likely to develop severe hypertension, eclampsia and obstetric hemorrhage [1]. In southern China, the carrier rate of $\alpha$-thalassaemia is $8.53 \%$ [2]. Among various genotypes, Southeast Asia deletion (-- ${ }^{\text {SEA })}$ is the most commonly observed, with incidence rates ranging from $72.87 \%$ to $82.87 \%$ [3, 4]. For this autosomal recessive disorder, carriers of one mutant alleleare asymptomatic, but couples who are both carriers of the -- ${ }^{\text {SEA }}$ mutation $(--\mathrm{SEA} / \alpha \alpha)$ run a $25 \%$ risk of having a hydropic fetus. For this reason, prenatal diagnosis in pregnancies has been advocated by the Chinese government for a number of years.

Preimplantation genetic diagnosis (PGD) is considered as an alternative for prenatal diagnosis. Many reports of successful PGD performed for $\beta$-thalassaemia have been published [5, 6]; however, PGD for $\alpha$-thalassaemia remains poorly studied [7-10]. To the best of our knowledge, only three live births have been reported $[9,10]$, including two by our group. The reason may be partly attributable to the fact that this regional disease is mainly found in economically less developed Southeast Asian countries. Previously, we established a fluorescent gap PCR protocol for 


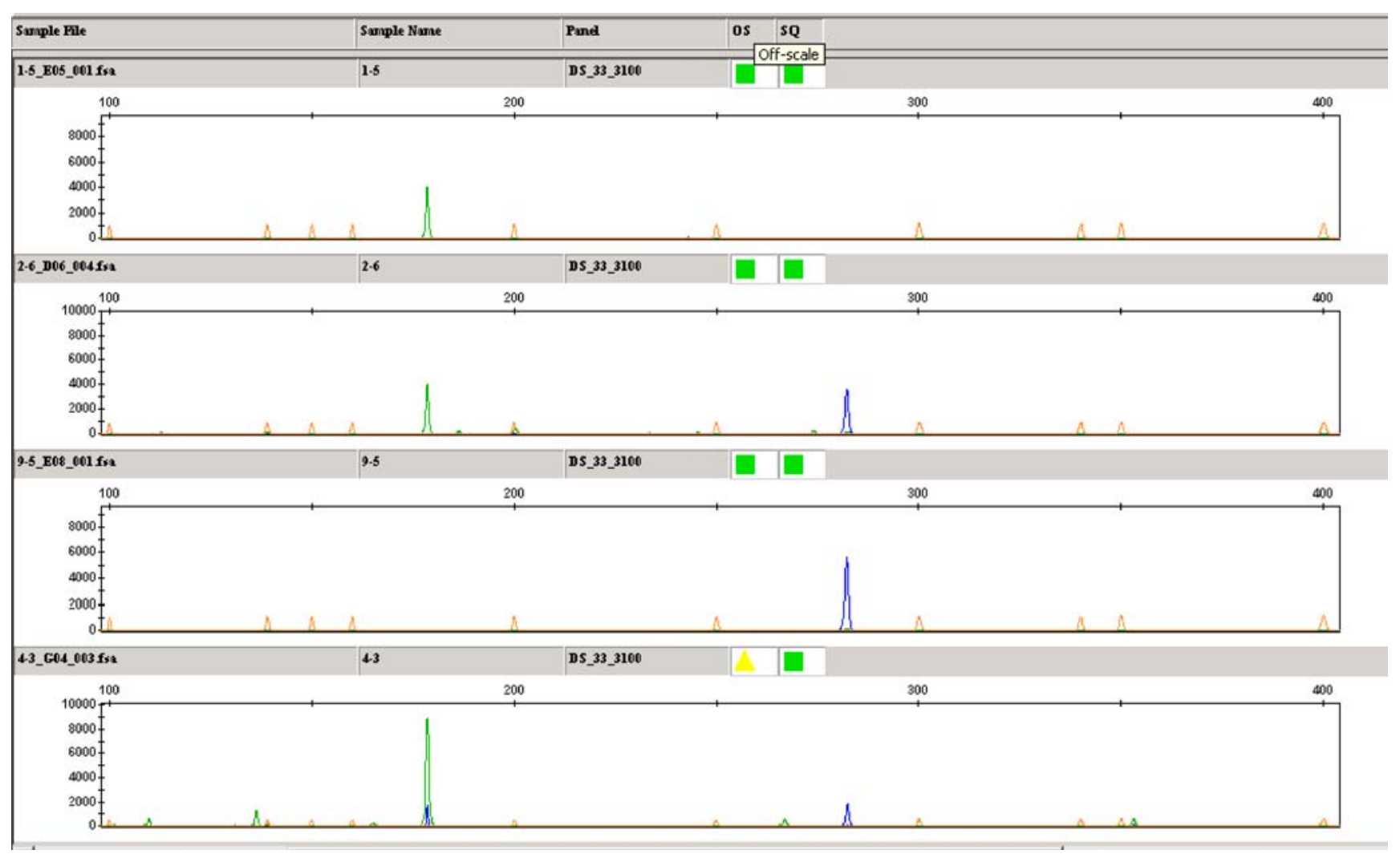

Fig. 1 Eletrophoretogram of PCR products for PGD. The top, second and third lanes are the affected, heterozygote, and normal samples, respectively. The bottom lane shows the heterozygote sample with preferential amplification in affected allele

the PGD of carriers with $\alpha$-thalassaemia in 2004 [9]. Here, we report our clinical application of 51 PGD cycles during a 4-year period.

\section{Materials and methods}

Patients

Between January 2004 and December 2007, 51 cycles of PGD were performed for 43 couples with the --SEA genotype (deletion of $2 \alpha$-globin genes in cis). Thirty-five couples underwent a single PGD cycle, and the remaining eight couples underwent two PGD cycles. Twenty-nine couples had a history of abnormal pregnancies. A total of 68 hydropic fetuses diagnosed by prenatal diagnosis as the --/-- genotype were selectively terminated in these couples. The subsequent analysis showed both parents were carriers of the -- SEA genotype (-- $\left.{ }^{\text {SEA }} / \alpha \alpha\right)$. In addition, fourteen couples without abnormal pregnancy history were infertile, and in each case both partners were diagnosed as carriers of the -- ${ }^{\text {SEA }}$ genotype during routine genetic screening prior to IVF in our center.

Ethnical approval was obtained for this study from the Ethnical Board of Sun Yat-sen University.
Methods

\section{Ovarian stimulation and embryo culture}

Ovarian stimulation was performed by a desensitizing long protocol using a gonadotrophin-releasing hormone analog (Daphiline Beaufour IPSEN, France) in association with recombinant follicle stimulating hormone (Gonal-F, Laboratories Serono SA, Switzerland) and human chorionic gonadotrophin (Profasi, Laboratories Serono SA, Switzerland). Oocyte retrieval was carried out by ultrasound-guided puncture $36 \mathrm{~h}$ after human chorionic gonadotrophin administration.

Standard intracytoplasmic sperm injection (ICSI) was performed on metaphase II oocytes [11]. After ICSI, oocytes were cultured in HTF medium (SAGE BioPharma, US) for 3 days.

\section{Embryo biopsy}

In PGD using blastomere biopsy, embryos with at least five blastomeres were biopsied in $\mathrm{Ca}^{2+} / \mathrm{Mg}^{2+}$ free medium under oil (SAGE, BioPharma, US) on the morning of day 3 after oocyte retrieval. Partial zona dissection (PZD) was performed to make a split in the zona pellucida. A sampling 
micropipette was pushed through the split into the zona to withdraw single cells. After biopsy, embryos were rinsed carefully and cultured in blastocyst medium (SAGE BioPharma, US) until transfer [9].

\section{$P G D$ for $\alpha$-thalassaemia}

The biopsied blastomeres were washed three times in sterile phosphate buffer saline (PBS) and transferred into a $0.2 \mathrm{~mL}$ Eppendorf tube containing a lysis solution of $0.5 \mu \mathrm{L}$ 10XPCR Buffer, $0.5 \mu \mathrm{L} 1 \%$ Tween-20, $0.5 \mu \mathrm{L}$ $1 \%$ Triton-100, $3.5 \mu \mathrm{L} \mathrm{H}_{2} \mathrm{O}$, and $0.05 \mu \mathrm{L}$ proteinase $\mathrm{K}$ $(20 \mathrm{mg} / \mathrm{mL})$ as described previously [9]. For each biopsied blastomere, a blank control was prepared from the final PBS cell wash drop. The positive controls were prepared with $10 \mathrm{pg}$ of purified DNA from the patients.

Biopsied blastomeres were lysed by incubating at $45^{\circ} \mathrm{C}$ for $15 \mathrm{~min}$ and inactivated by heating to $96^{\circ} \mathrm{C}$ for $20 \mathrm{~min}$. The amplification involved the use of three $\alpha$-thalassaemia SEA primers. The S1 and S3 primers flank the SEA deletion, while the S2 primer anneals within the deleted area [12]. The sequence of S1 is 5'-gtgttctcagtattggagggaa-3'. The S2 and S3 primers had the sequence of $5^{\prime}$-FAM-gacacgettccaatacgctta- $3^{\prime}$ and $5^{\prime}$-HEX-ctactgcagccttgaactcc-3', and were labeled with blue and green fluorescent dye, respectively. In wild-type samples, the S1 and S2 primers amplified a product of $282 \mathrm{bp}$. However, in mutant samples, the S1 and S3 are brought into close proximity by deletion of the intervening sequence and were able to amplify a product of $178 \mathrm{bp}$ (Fig. 1).

Aliquots of $50 \mu \mathrm{l}$ PCR master mix containing $5 \mu \mathrm{l}$ lysis solution, 4.5 $\mu \mathrm{l}$ 10XPCR neutralizing buffer, $1.5 \mu \mathrm{l}$ $50 \mathrm{mmol} / \mathrm{L} \mathrm{MgCl} 2,1 \mu \mathrm{l}$ each $10 \mathrm{mmol} / \mathrm{L}$ dNTP, $2.0 \mu \mathrm{l}$ each of primers $\mathrm{S} 1, \mathrm{~S} 2$ and $\mathrm{S} 3,1.5 \mathrm{U}$ AmpliTap DNA polymerase $(\mathrm{ABI})$ were added to reaction tubes. PCR reactions were performed using a Perkin Elmer Cetus 9700 PCR machine. The program used was $2 \mathrm{~min}$ initial denaturation at $96^{\circ} \mathrm{C}$ followed by $45 \mathrm{~s}$ denaturation at $96^{\circ} \mathrm{C}$ for the first 10 cycles and $94^{\circ} \mathrm{C}$ for the 40 remaining cycles. Annealing and elongation cycles were performed for $45 \mathrm{~s}$ at $60^{\circ} \mathrm{C}$ and $60 \mathrm{~s}$ at $72^{\circ} \mathrm{C}$, respectively. The PCR reaction was terminated by $7 \mathrm{~min}$ at $72^{\circ} \mathrm{C}$. PCR products were then analyzed on an ABI 3100 Advant genetic analyzer.

Only embryos diagnosed as wild-type or heterozygous would be recommended as suitable for embryo transfer. Following genetic diagnosis, one to three suitable embryos were selected on morphological criteria and transferred on day 4 with cryopreservation of any remaining suitable embryos.

\section{Results}

A total of 51 biopsy cycles were carried out for 43 couples, with an average age of the female partner at $30.7 \pm 3.5$. An average of 17.3 oocytes was retrieved per cycle with a fertilization rate of $70.9 \%$.

The embryo biopsy procedure was successfully performed in all cases. A total of 472 embryos were biopsied, of which 390 were successfully amplified with an amplification rate of $82.6 \%$. Diagnosis of 36 embryos yielded ambiguous results: 24 showed nonspecific amplification products along with target gene products, and eight exhibited contamination in blank controls. Overall, 120 normal homozygous (wild-type) embryos, 94 heterozygous carrier embryos and 140 abnormal homozygous embryos were diagnosed respectively, accounting for a total diagnostic rate of $75.0 \%$ (354/472).

Preferential amplification was detected in 18 blastomeres. In the re-analysis of 49 blastomeres from six discarded heterozygous embryos, 39 blastomeres were successfully amplified, of which 9 showed allele drop-out (ADO), indicative of an ADO rate of $16.4 \%[9 /(49+6)]$.

A total of 154 embryos including 101 wild-type embryos and 53 heterozygous embryos were transferred, resulting in 25 clinical pregnancies with an implantation rate of $24.0 \%$ (37/154). The 25 clinical pregnancies included 12 singletons, six twins, four triplets and one tubal pregnancy. Two singleton miscarriages occurred before 12 weeks of pregnancy. One fetus was diagnosed as a 13 trisomy by prenatal diagnosis, and selective termination was performed at 20 weeks of gestation. Spontaneous fetal reduction occurred in one twin and one triplet pregnancies. Fetal reduction was performed in the three remaining triplets according to the official regulation. After fetal reduction, routine ultrasound at 13 weeks of gestation revealed one case suffered fetal edema in one of the two remaining fetuses; consequently, a second fetal reduction was performed. The chromosomal analysis of fetal tissue showed a 45,XO karyotype. In summary, 27 healthy babies were born. Prenatal diagnosis showed 17 were wild-type and 10 were heterozygous for the $\alpha$-globin loci. No misdiagnosis was observed.

\section{Discussion}

Prenatal diagnosis for $\alpha$-thalassaemia has been proven to be an effective strategy for combating severe obstetric complications in mothers carrying hydropic fetus in late gestation and is widely used in southeast China. However, once diagnosed, the decision to terminate an affected pregnancy is sometimes difficult. Furthermore, repeated termination of affected pregnancies will also cause both physical and mental trauma to mothers. In this study, a total of 68 hydropic fetuses were selectively terminated in 29 couples. The establishment of a PGD protocol offers an opportunity to avoid the adverse consequences of pregnancy termination, since abnormal embryos 
will not be transferred. In addition, couples suffering from fertility problems may consider transfer unaffected embryos by PGD when receiving IVF treatment.

Gap PCR is a reliable method for prenatal diagnosis of $\alpha$-thalassaemia [12]. Previously, semi-nested or nested second round PCR has been adopted in the PGD for $\alpha$-thalassaemia to ensure specific amplification [7]. The detection sensitivity for small amounts of PCR products was improved by the application of fluorescent labeled primers and the analysis of amplified PCR products by a genetic analyzer. In the study by Chan et al. [10], multiplex fluorescent gap-PCR was performed to include 34 amplification cycles in the first round and 23 cycles in the second round PCR. Moreover, they estimated the ADO and PCR failure rate at $10.2 \%$ and $12.7 \%$, respectively, lower than the $24.9 \%$ reported previously [7].

In the present study, the use of single-round fluorescent PCR with 50 cycles was sufficient to amplify PCR products for the analysis on the genetic analyzer. The ADO and PCR failure rate was $16.4 \%$ and $17.4 \%$, respectively, only slightly higher than those from two-round PCR by Chan et al. [10]. Furthermore, single-round fluorescent PCR significantly reduces the risk of contamination and is much less laborious to perform.

$\mathrm{ADO}$ and PCR failure rates are the main parameters for the evaluation of feasibility of a PGD strategy. ADO is considered as the main cause of misdiagnosis in PGD [13, 14]. In the current study, the ADO rate was estimated from the re-analysis of discarded embryos. However, chromosome mosaicism in discarded embryos may affect the ADO rate. In fact, the ADO rate can be deduced from the ratio of heterozygote embryos. Only $26.6 \%$ (94/354) embryos were diagnosed as the heterozygote embryo, which was significantly lower than the expected ratio of $50 \%$. In the case of heterozygote embryos, ADO for the normal allele may lead to misdiagnosis as the homozygous mutant embryo; conversely, ADO of the mutant allele may cause misdiagnosis as the normal homozygote embryo. Collectively, such diagnostic deficiencies will lead to a lower ratio of heterozygote embryos and a reduced number of embryos available for transfer in the present study. Nevertheless, since $\alpha$ thalassaemia is an autosomal recessive disease, the presence of the normal allele in the transferred embryos should offset any adverse consequence due to ADO in the mutant allele.

Contamination in single-cell PCR from exogenous DNA can not be reliably excluded with currently available methods [15]. To minimize such risk, we employed dedicated airborne manipulator for PGD, and the biopsy area for clinical application was physically separated from tubing and pre-PCR areas. Post-PCR analysis was performed in a distinct space from pre-PCR steps. Blastomere tubing and PCR preparation were carried out in a clean laminar flow cabinet. Although eight blastomeres exhibited contamination in blank controls and 24 showed nonspecific amplification products along with target gene products in the present study, the vast majority of reactions produced clear results devoid of any contamination.

In summary, single-round fluorescent gap PCR is a feasible and effective strategy in the PGD for $\alpha$-thalassaemia. However, the ADO and PCR failure rates remain as major concerns for diagnostic accuracy and efficiency. Analogous to the PGD for Duchenne muscular dystrophy via multiple displacement amplification recently published by our group [16], the closely linked STR locus will be analyzed in parallel in future studies to reduce the risk of misdiagnosis and omission of heterozygotes due to ADO in the normal allele. Finally, blastocyst biopsy, which provides more trophectoderm cells for diagnosis, will also be considered to overcome the inherent sensitivity limitation of single-cell PCR [17].

Acknowledgements This work was supported by National Basic Research Program of China (grant no. 2007CB948101), China Medical Board of New York Inc (grant No 06840) and National Natural Science Foundation of China (grant no. 30700910).

Declaration The authors report no financial or commercial conflicts of interest.

Open Access This article is distributed under the terms of the Creative Commons Attribution Noncommercial License which permits any noncommercial use, distribution, and reproduction in any medium, provided the original author(s) and source are credited.

\section{References}

1. Liang ST, Wong VCW, So WWK, Ma HK, Chan V, Todd D. Homozygous $\alpha$-thalassaemia: clinical presentation, diagnosis and management. A review of 46 cases. Br J Obstet Gynaecol. $1985 ; 92: 680-4$.

2. Xu XM, Zhou YQ, Luo GX, et al. The prevalence and spectrum of alpha and beta thalassaemia in Guangdong Province: implications for the future health burden and population screening. J Clin Pathol. 2004;57:517-9.

3. Huang YJ, Ou XB, Yu YP, et al. The incidence rates and genotypes in children with $\alpha$-thalassaemia in Guangzhou region. Chin Pedi Hemat. 2005;10:205-8.

4. Duan S, Li HY, Chen Z, et al. The research on the mutation genotypes of $\alpha$-thalassaemia in Southern China. Zhong-Guo-ShiYan-Xue-Ye-Xue-Za-Zhi. 2003;11:54-60.

5. Kanavakis E, Vrettou C, Palmer G, et al. Preimplantation genetic diagnosis in 10 couples at risk for transmitting beta-thalassemia major: Clinical experience including the initiation of six singleton pregnancies. Prenat Diagn. 1999;19:1217-1222.

6. De Rycke M, Van de Velde H, Sermon K, et al. Preimplantation genetic diagnosis for sickle cell anemia and fro beta-thalassemia. Prenat Diagn. 2001;21:214-222.

7. Chang MY, Soong YK, Wong ML. Preimplantation diagnosis of $\alpha$ thalassemia by blastomere aspiration and polymerase chain reaction: preliminary experience. J Formos Med Assoc. 1996;95:203-208.

8. Piyamongkol W, Harper JC, Delhanty J, et al. Preimplantation genetic diangositic protocols for $\alpha$ - and $\beta$-thalassaemias using multiplex fluorescent PCR. Prenat Diagn. 2001;21:753-759. 
9. Deng J, Peng WL, Li J, et al. Successful preimplantation genetic diagnosis for alpha- and beta-thalassemia in China. Prenat Diagn. 2006;26:1021-8.

10. Chan V, Ng E, Yam I, et al. Experience in preimplantation genetic diagnosis for exclusion of homozygous $\alpha^{0}$ thalassemia. Prenat diagn. 2006;26:1029-36.

11. Palermo G, Joris H, Devroey P, Van Steirteghem AC. Pregnancies after intracytoplasmic injection of single spermatozoan into an oocyte. Lancet. 1992;340:17-8.

12. Ko TM, Tseng LH, Hsieh FJ, et al. Carrier detection and prenatal diagnosis of alpha-thalassemia of Southest Asian deletion by polymerase chain reaction. Hum Genet. 1992;88:245248.

13. Findlay I, Ray P, Quirke P, et al. Allelic dropout and preferential amplification in single cells and human blastomeres: implication for preimplantation diagnosis of sex and cystic fibrosis. Hum Reprod. 1995;10(6):1609-18.

14. Wells D, Sherlock J. Diagnosis of single gene disorder. In: Harper J, Delhanty J, Handyside AH, editors. Preimplantation genetic diagnosis. England: Wiley; 2000. p. 165-190.

15. Moutou C, Gardes N, Rougiéres C, et al. Allele-specific amplification for preimplantation genetic diagnosis of spinal muscular atrophy. Prenat Diagn. 2001;21:498-503.

16. Ren Z, Zeng HT, Xu YW, et al. Preimplantation genetic diagnosis for Duchenne muscular dystrophy by multiple displacement amplification. Fertil Steril. 2009;91:359-64.

17. Kokkali G, Traeger-Synodinos J, Vrettou C, et al. Blastocyst biopsy versus cleavage stage biopsy and blastocyst transfer for preimplantation genetic diagnosis of $\beta$-thalassaemia: a pilot study. Hum Reprod. 2007;22:1445-9. 